\title{
Why engineering laws have been irrational for more than 70 years, and rational laws to replace them.
}

\author{
Eugene F. Adiutori \\ efadiutori@aol.com \\ 239-537-4107 \\ Ventuno Press \\ 1094 Sixth Lane North \\ Naples, FL 34102 \\ December 15, 2021
}

\begin{abstract}
In order to confirm that conventional engineering laws are rational, it is necessary to know how the coefficients in the laws were created. Until 1822, scientists and engineers agreed that equations cannot describe how parameters are related because parameter dimensions cannot be multiplied or divided. (That is why Newton's second law of motion in The Principia (1726) is not an equation - it is a proportion.) In The Analytical Theory of Heat (1822), Fourier made the revolutionary and unproven claims that dimensions can be assigned to numbers, and parameter dimensions can be multiplied and divided. These unproven claims made it rational for Fourier to create $h$ by assigning the dimensions of $q / \Delta T$ to number $c$ in his empirically determined proportional equation $q=c \Delta T$, and rational to multiply $h$ (ie $c$ with assigned dimensions) and $\Delta T$, resulting in dimensionally homogeneous $q=h \Delta T$. After 1822, engineering laws were generally created using Fourier's unproven claims. However, Dimensional Analysis and Theory of Models by H. L. Langhaar (1951) states: Dimensions must not be assigned to numbers, for then any equation could be regarded as dimensionally homogeneous. Because conventional engineering laws were generally created by violating the widely accepted view that dimensions must not be assigned to numbers, laws such as $q=h \Delta T, \sigma=E \varepsilon, E=I R$ became irrational sometime before 1951. Rational engineering laws determined by induction are presented herein.
\end{abstract}

\section{Introduction}

In order to confirm that conventional engineering laws are rational, it is necessary to know how the coefficients in the laws were created. Fourier [1] created the coefficient $h$, and the methodology he used to create $h$ has generally been used to create coefficients in similar engineering laws.

Fourier's methodology requires that dimensions be assigned to numbers. However, sometime before 1951, it was generally agreed that dimensions cannot rationally be assigned to numbers. Therefore engineering laws created by assigning dimensions to numbers have been irrational since sometime before 1951.

Rational engineering laws determined by induction are presented herein. 


\section{Parametric equations from the beginning of science until 1822 .}

From the beginning of science until 1822 , scientists and engineers globally agreed that equations cannot rationally describe how parameters are related because parameter dimensions cannot rationally be multiplied and divided. Proportions are generally used instead of equations because they do not require that parameter dimensions be multiplied or divided. That is why Newton's second law of motion in The Principia [2] is not Eq. (1). It is Proportion (2).

$f=m a$

$a \alpha f$

\section{Fourier's experiment, and the dimensionally inhomogeneous equation that resulted.}

Fourier performed an experiment in heat transfer by the steady-state forced convection of ambient air flowing over a solid, warm body. From his data, he concluded that if a solid, warm body is cooled by the steady-state forced convection of ambient air, the heat flux from the surface of the solid, warm body to the ambient air is always proportional to the temperature difference between the surface of the solid, warm body and the ambient air, as in Proportion (3).

$q \alpha \Delta T$

Proportion (3) would have satisfied Newton and his colleagues, but it did not satisfy Fourier. He wanted an equation, and it had to be dimensionally homogeneous.

The transformation of Proportion (3) to an equation results in Eq. (4). Note that Eq. (4) is a proportional equation, and $c$ is a number that equals the numerical value of $q / \Delta T$.

$q=c \Delta T$

Fourier was not satisfied with Eq. (4) because it is not dimensionally homogeneous - the dimension of $q$ does not equal the dimension of $\Delta T$, and $c$ is dimensionless. Fourier recognized that Eq. (4) could be made dimensionally homogeneous only if it were rational to assign dimensions to numbers, and rational to multiply and divide parameter dimensions.

\section{How Fourier created h.}

In The Analytical Theory of Heat published in 1822, Fourier made the following revolutionary and unproven claims:

- Dimensions can be assigned to numbers.

- Parameters can be multiplied and divided because parameter dimensions can be multiplied and divided.

Fourier described his revolutionary and unproven claims in the following paragraph: 
... every undetermined magnitude or constant has one dimension proper to itself, and the terms of one and the same equation could not be compared, if they had not the same exponent of dimension. . . this consideration is derived from primary notions on quantities; for which reason, in geometry and mechanics, it is the equivalent of the fundamental lemmas (axioms) which the Greeks have left us without proof.

It is important to note that the above paragraph has been the foundation of conventional engineering science for 200 years. It is the only reason it has been rational to create laws by assigning dimensions to numbers, and rational to multiply and divide parameter dimensions (in violation of the view that prevailed from the beginning of science until 1822).

It is also important to note that Fourier made no effort to prove that his revolutionary claims are rational. In his nearly 500 page treatise, he did not include the Greek lemmas, he did not cite a reference where the lemmas could be found, and he did not offer his own proof that his revolutionary claims are rational.

Fourier's revolutionary and unproven claims made it rational to transform dimensionally inhomogeneous Eq. (4) to dimensionally homogeneous Eq. (5) by making it rational to assign to pure number $c$ the dimensions of $q / \Delta T$ in order to make Eq. (5) dimensionally homogeneous, and rational to multiply $h$ (ie $c$ with dimensions) and $\Delta T$.

$q=h \Delta T$

Equation (5) should be referred to as Fourier's law of steady-state forced convection heat transfer to ambient air. It is a proportional equation, and it applies only if heat transfer is by the steadystate forced convection of ambient air flowing over a solid, warm body. To Fourier and his colleagues, Eq. (5) meant that, if heat transfer is by the steady-state forced convection of ambient air flowing over a solid, warm body:

- The numerical value and dimension of $q$ are always proportional to the numerical value and dimension of $\Delta T$.

- $h$ and $q / \Delta T$ are always identical and interchangeable because $c$ in Eq. (4) is numerically equal to $q / \Delta T$, and $c$ was assigned the dimension of $q / \Delta T$.

- $h$ and $q / \Delta T$ are always proportionality constants - ie they are never variables. In other words, Eq. (5) always has only two variables, $q$ and $\Delta T$.

- Equation (5) is always dimensionally homogeneous because the dimension of $q$ always equals the dimension of $h \Delta T$ - ie the dimension of $q$ always equals the dimension of $(q / \Delta T) \Delta T$.

In summary, Fourier was able to create the very first dimensionally homogeneous engineering law because of his revolutionary and unproven claims that dimensions can be assigned to numbers, and parameter dimensions can be multiplied and divided. Later engineering laws were generally created using Fourier's unproven claims. 


\section{Why Fourier's claim that dimensions can be assigned to numbers is irrational.}

Page 13 of Dimensional Analysis and Theory of Models by H. L. Langhaar published in 1951 states:

Dimensions must not be assigned to numbers, for then any equation could be regarded as dimensionally homogeneous.

Fourier's pioneering method of assigning dimensions to numbers is irrational because it assumes that dimensions can rationally be assigned to numbers, when in fact it has been widely agreed for more than 70 years that dimensions must not be assigned to numbers.

\section{Why Fourier's claim that parameter dimensions can be multiplied and divided is irrational.}

From the beginning of science until 1822, scientists and engineers such as Galileo and Newton agreed that equations cannot describe how different parameters are related because parameter dimensions cannot be multiplied or divided. Fourier claimed, without proof, that parameter dimensions can rationally be multiplied or divided. Note that:

- "Multiply five times eight" means "add eight five times". Therefore "multiply meters times kilograms" must mean "add kilograms meters times". Because "add kilograms meters times" has no meaning, parameter dimensions cannot rationally be multiplied.

- "Divide thirty by six" means "how many sixes are in thirty". Therefore "divide meters by seconds" must mean "how many seconds are in meters". Because "how many seconds are in meters" has no meaning, dimensions cannot rationally be divided.

In summary, parameters cannot be multiplied or divided because parameter dimensions cannot be multiplied or divided, in accordance with the view that prevailed from the beginning of science until 1822.

\section{Why the laws and equations of modern engineering science do not rationally describe how parameters are related.}

The laws and equations of modern engineering science do not rationally describe how parameters are related because they are based on the erroneous assumptions that dimensions can rationally be assigned to numbers, and parameter dimensions can rationally be multiplied and divided.

It is important to note that even if a law in the form of a proportional equation were rational, it could not describe nonlinear phenomena. When a proportional equation is applied to nonlinear phenomena, it ceases to be an equation because it cannot describe how parameters are related if the phenomenon is nonlinear.

For example, Eq. (5) is a proportional equation and therefore it cannot describe the nonlinear behavior of heat transfer by natural convection, condensation, or boiling. When Eq. (5) is 
applied to a nonlinear phenomenon, it ceases to be an equation because it makes no statement about the relationship between $q$ and $\Delta T$. It merely states the following:

To determine the value of $q$, first determine the value of $q / \Delta T$ (ie the value of $h$ ), then multiply by $\Delta T$.

\section{What equations can rationally describe about how parameters are related.}

Equations can rationally describe how the numerical values of parameters are related because, if parameter symbols represent only numerical values, equations do not require that dimensions be assigned to numbers, and do not require that parameter dimensions be multiplied and divided.

\section{The rational law of stress and strain.}

Data indicate that the numerical value of stress is a function of the numerical value of strain, and the function may be proportional, linear, or nonlinear. Therefore Eq. (6) is the rational law of stress and strain.

$\sigma=f\{\varepsilon\}$

$\varepsilon=f\{\sigma\}$

Equation (6a) states that the numerical value of stress is a function of the numerical value of strain, and the function may be proportional, linear, or nonlinear. And similarly for Eq. (6b).

Note that $E$ was created for only one reason-so that the law of stress and strain could be dimensionally homogeneous. Because Eq. (6) is inherently dimensionally homogeneous, $E$ is not required for homogeneity, and is abandoned. Abandoning $E$ greatly simplifies the solution of inelastic problems because, in the inelastic region, $E$ is an unnecessary and undesirable variable, and it greatly complicates problem solutions.

\section{The rational law of convection heat transfer.}

Data indicate that, if heat transfer is by convection, the numerical value of heat flux is a function of the numerical value of temperature difference, and the function may be proportional, linear, or nonlinear. Therefore Eq. (7) is the law of convection heat transfer.

$q=f\{\Delta T\}$

$\Delta T=f\{q\}$

Equation (7a) states that the numerical value of heat flux is a function of the numerical value of temperature difference, and the function may be proportional, linear, or nonlinear. And similarly for Eq. (7b). 
Note that $h$ was created for only one reason-so that the law of convection heat transfer could be dimensionally homogeneous. Because Eq. (7) is inherently dimensionally homogeneous, $h$ is not required for homogeneity, and is abandoned. Abandoning $h$ greatly simplifies the solution of nonlinear problems because $h$ is an unnecessary and undesirable variable if $q$ is a nonlinear function of $\Delta T$.

\section{An analog of rational engineering laws.}

Equation (8) is an analog of rational engineering laws.

$y=f\{x\}$

Equation (8) states that the numerical value of parameter $y$ is a function of the numerical value of parameter $x$, and the function may be proportional, linear, or nonlinear.

12. Quantitative equations when engineering laws are analogs of Eq. (8).

If an equation is quantitative and engineering laws are analogs of Eq. (8), the dimension units that underlie parameter symbols must be specified in an accompanying nomenclature. This applies only to parameter symbols that are not in dimensionless groups.

\section{How textbooks must be transformed when analogs of Eq. (8) replace conventional engineering laws.}

When analogs of Eq. (8) replace conventional engineering laws, textbooks must be transformed by eliminating parameters such as $h$ and $E$-ie by eliminating $q / \Delta T$ and $\sigma / \varepsilon$. For example, a heat transfer textbook is transformed in the following manner:

- Note that, because $q=h \Delta T, h$ and $q / \Delta T$ are identical and interchangeable.

- Note that $k / t=q / \Delta T$.

- In all equations, replace $h$ and $k / t$ by $q / \Delta T$, even if $h$ or $k / t$ is in a dimensionless group such as Nusselt number. Note that Nusselt number is abandoned because it contains $h$-ie it contains $q / \Delta T$.

- Separate $q$ and $\Delta T$, resulting in equations in the form of Eq. (9).

$$
\Delta T=f\{q\}
$$

For example, when $q$ and $\Delta T$ are separated, Eq. (10) is transformed to Eq. (11).

$$
\begin{aligned}
& U=\left(1 / h_{1}+t / k+1 / h_{2}\right)^{-1} \\
& \Delta T_{\text {total }}=\Delta T_{1}\{q\}+\Delta T_{\text {wall }}\{q\}+\Delta T_{2}\{q\}
\end{aligned}
$$


Note that Eqs. (10) and (11) are identical - they state exactly the same thing. When Eq. (7) replaces Eq. (5), Eq. (11) replaces Eq. (10). And similarly for E.

\section{Conclusions}

- Dimensions cannot rationally be assigned to numbers.

- Parameters cannot rationally be multiplied or divided because parameter dimensions cannot be multiplied or divided.

- Conventional engineering laws are irrational because they are based on the erroneous assumptions that dimensions can be assigned to numbers, and parameter dimensions can be multiplied and divided.

- Because parameter dimensions cannot be multiplied or divided, parameter symbols in equations must represent only numerical values.

- Data indicate that Eq. (6) is a rational law of stress and strain. Equation (6a) states that the numerical value of stress is a function of the numerical value of strain, and the function may be proportional, linear, or nonlinear. Similarly for Eq. (6b).

$$
\begin{aligned}
& \sigma=f\{\varepsilon\} \\
& \varepsilon=f\{\sigma\}
\end{aligned}
$$

- Data indicate that Eq. (7) is a rational law of convection heat transfer. Equation (7a) states that the numerical value of heat flux is a function of the numerical value of temperature difference, and the function may be proportional, linear, or nonlinear. Similarly for Eq. (7b).

$q=f\{\Delta T\}$

$\Delta T=f\{q\}$

- Equation (8) is an analog of rational engineering laws. Equation (8) states that the numerical value of parameter $y$ is a function of the numerical value of parameter $x$, and the function may be proportional, linear, or nonlinear.

$y=f\{x\}$

- When conventional engineering laws are replaced by analogs of Eq. (8), parameters such as $h$ and $E$ are unnecessary and undesirable, and they are abandoned.

- If an equation is quantitative and engineering laws are analogs of Eq. (8), the dimension units that underlie parameter symbols must be specified in an accompanying nomenclature. This applies only to parameter symbols that are not in dimensionless groups. 
- If engineering laws are analogs of Eq. (8), textbooks must be transformed so that parameters such as $h$ and $E$ are eliminated. For example, a heat transfer text is transformed by substituting $q / \Delta T$ for $h$ and for $k / t$, then separating $q$ and $\Delta T$ to result in equations in the form $\Delta T=f\{q\}$. Similarly for $E$ and other coefficients in engineering laws that are in the form of proportional equations.

\author{
Symbols \\ a acceleration \\ c arbitrary constant \\ $f \quad$ force or function of \\ $h \quad$ symbol for $q / \Delta T$ \\ $k \quad$ symbol for $q /(d T / d x)$ \\ $m$ mass \\ $q$ heat flux \\ $t \quad$ time \\ $T$ temperature \\ $U \quad$ symbol for $q / \Delta T_{\text {total }}$
}

\title{
References
}

[1] Fourier, J., 1822d, The Analytical Theory of Heat, p. 2 of Preliminary Discourse and Article 160, 1955 Dover edition of 1878 English translation

[2] Newton, I., The Principia, 1726, $3^{\text {rd }}$ edition, translation by Cohen, I. B. and Whitman, A. M., 1999, p. 460, University of California Press 